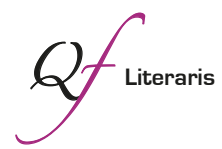

\title{
La traducción como vehículo de divulgación de la doctrina espiritista: el caso de Defensa del espiritismo de A.R. Wallace
}

\author{
Translation as a vehicle for dissemination of the spiritualist doctrine: \\ the case of Defensa del espiritismo by A.R. Wallace
}

\section{M. ${ }^{a}$ del Mar Verdejo Segura}

Universidad de Málaga. verdejo@uma.es

Recibido: 09/05/2017. Aceptado: 14/11/2017

Resumen: En la segunda mitad del siglo XIX España fue testigo del desarrollo y apogeo del movimiento espiritista el cual trajo consigo un impulso de la actividad traslativa. En este trabajo nos centraremos en la traducción del libro de A.R. Wallace On Miracles and Modern Spiritualism, obra de referencia en la difusión de la doctrina espiritista que fue publicada en nuestro país en 1891 con el título Defensa del espiritismo. Fijaremos nuestra atención en los paratextos que acompañan a la traducción y destacaremos los procedimientos de traducción más relevantes empleados por el traductor, Alfonso Herrera Fernández.

Palabras clave: Espiritismo; traducción; Defensa del espiritismo; A.R.Wallace

\begin{abstract}
The second half of nineteenth-century Spain witnessed the growth and development of the spiritualist movement, which stimulated translation. This article focuses on A.R. Wallace's seminal work On Miracles and Modern Spiritualism, which played a crucial role in the dissemination of this doctrine. Published in Spain in 1891 under the title Defensa del espiritismo, the book's paratexts and the translation procedures employed by the translator, Alfonso Herrera Fernández, will be here analysed.
\end{abstract}

Keywords: Spiritualism; translation; Defensa del espiritismo; A.R.Wallace. 



\section{Introducción}

En la segunda mitad del siglo XIX España, al igual que otros países, fue testigo del desarrollo y apogeo del movimiento espiritista ${ }^{1}$, el cual conllevó, entre otras muchas acciones, un estímulo de la actividad traslativa por parte de los adeptos a esta doctrina en su tarea divulgativa. El estudio de la actividad traductora llevada a cabo por éstos, así como por representantes de otros grupos marginales, tales como los teósofos o los protestantes, ha recibido, que sepamos, poca atención por parte de los investigadores ${ }^{2}$. Sin embargo, dada la magnitud de los textos traducidos y el alcance que algunos de ellos tuvieron, esta situación de descuido consideramos que está injustificada y que merece mayor atención. En este sentido coincidimos con lo expresado por (Bastin, 2011: 36): "¿Qué hay de otras latitudes y qué pasa con la traducción no literaria? La historia de la traducción debe abarcar una visión amplia sin excluir campo alguno de la actividad humana".

En este trabajo, desde el paradigma de la Traductología, nos centraremos en la traducción del libro de A.R. Wallace On Miracles and Modern Spiritualism (1875)³, publicado en nuestro país en 1891 con el título de Defensa del espiritismo. La elección de este libro se debe a varios motivos fundamentales. Fue, y continúa siendo, una obra de referencia sobre el tema muy empleada en la difusión de la doctrina espiritista. Prueba de ello es que ya en el siglo XIX el texto había sido traducido a varios idiomas, en ocasiones, como en el caso alemán, incluso en el mismo año de la publicación del original. Es, además, una de las obras de procedencia anglosajona traducidas sobre el tema que nos ocupa. Ello es relevante ya que en nuestro país la influencia del espiritismo francés, representado en la figura de Kardec, es incuestionable 4 .

\footnotetext{
* Este estudio deriva de la investigación realizada en el marco del Proyecto de investigación I+D financiado por el Ministerio de Economía y Competitividad La traducción de clásicos en su marco editorial: Una visión transatlántica (FFI2013-41743-P).

${ }^{1}$ Entre las monografías que versan sobre el tema queremos destacar por su rigor y enfoque las De Mateo Avilés (2011) y Mülberger (2016).

${ }^{2}$ Los trabajos de Penalva Mora (2013), Verdejo Segura (2011) y Zaro Vera (2015) constituyen algunas excepciones.

${ }^{3}$ Antes de concluir el siglo la obra gozó en Gran Bretaña de dos ediciones más: una en 1881, y otra en 1896. Esta última revisada y ligeramente aumentada.

4 “Aunque se puede constatar también en España la influencia esporádica del espiritismo anglosajón (llamado spiritualism) fue sobre todo el espiritismo francés el que se configuró como movimiento social y religión laica", Mülberger (2016: 90).
} 
La influencia del autor francés, no obstante, se acusó también en otros países europeos e hispanoamericanos entre los que cabe mencionar, por su vínculo histórico con el país galo, México. A las razones expuestas hay que añadir esta otra: es, a nuestro entender, la única obra completa del autor británico traducida a nuestro idioma en el siglo XIX en España. Ello ha despertado nuestro interés porque, paradójicamente, no se trata de una de las muchas obras que lo encumbraron como científico sino que, por el contrario, fue, y así figura en la literatura sobre el autor, una causa determinante de su posterior desprestigio entre la élite científica del momento.

\section{Sobre el autor y la obra}

Alfred Russel Wallace (1823-1913), descrito como gran naturalista, biólogo, geógrafo, antropólogo, reformador, explorador y activo espiritista, es considerado uno de los intelectuales británicos más sobresalientes del siglo XIX.

Como científico, ocupación a la que se consagró la mayor parte de su vida, destaca por ser responsable de numerosos trabajos que contribuyeron al desarrollo de distintos campos del saber. Entre ellos cabe citar The Malay Archipelago (1869), un best-seller en la época, The Geographical Distribution of Animals (1876) o Man's Place in the Universe (1903). Irrebatible es su muy meritoria contribución a la teoría de la evolución ya que, según sostienen reputados estudiosos, fue artífice junto con Darwin de la teoría de la evolución por selección natural, atribuida tradicionalmente y de manera equivocada, únicamente a este último 5 .

Al margen de la ciencia, otras materias por las que el británico se interesó y a las que dedicó parte de su tiempo fueron la economía, la política y el espiritismo. El autor, como adepto que fue a la doctrina, estaba convencido de que el espiritismo ofrecía una respuesta que la ciencia no podía darle. Así, en el prólogo al libro, afirma: "this doctrine will enable us to account for some of those residual phenomena which Natural Selection alone will not explain" (p.vii) ${ }^{6}$. Berry (2013b:

\footnotetext{
${ }^{5}$ Véase Revets (2009), Costa (2014) y Ginnobily y Blanco (2017).

${ }^{6}$ Las citas del texto proceden de la edición de la obra publicada en Londres por James Burns en 1875. El volumen se encuentra disponible en: https://archive.org/details/onmiraclesmodern00wall.
} 
R1068), por su parte, señala al respecto que Wallace "saw spiritualism as a legitimate domain of scientific inquiry ${ }^{7}$. El ciéntifico británico fue también un prolífico autor. En su faceta de escritor, Smith (2009:35) lo describe como "a very effective wordsmith", a lo que añade: "Beyond merely being readable, moreover, Wallace was by all evidence effective at expressing just what he wanted to".

Por lo que respecta a la obra que nos ocupa, On Miracles and Modern Spiritualism, el volumen lo conforman tres ensayos que habían sido difundidos previamente. El primero, "An Answer to the Arguments of Hume, Lecky, and Others against Miracles", fue escrito con la intención de inducir a los escépticos a reconsiderar la credibilidad de los milagros. El segundo, "The Scientific Aspects of the Supernatural", pretende explicar los principios éticos fundamentales del espiritismo. En el mismo, el científico británico nos describe sus primeras incursiones en la materia y el impacto que las mismas le produjeron (p. 334). El tercero, "A Defence of Modern Spiritualism", ofrece datos sobre la historia del espiritismo.

\section{Traducciones de la obra al español anteriores a la edición española}

A modo de preámbulo a nuestros comentarios sobre la traducción empleada en la edición española, objeto central de este trabajo, consideramos oportuno señalar, pues de ello se informa en distintas páginas de dicha edición, que la obra de Wallace, antes de ser publicada en España había sido traducida a nuestro idioma en tierras hispanoamericanas. De hecho, el texto empleado en la edición española es una traducción publicada en México.

La primera traducción de la obra de la que tenemos noticia data de 1877 y se publicó en Santiago de Cuba. Años después, en 1887, el Centro de Propaganda Espiritista de Buenos Aires la publicaría de nuevo para su difusión gratuita con el título Defensa del Espiritismo. Se trata de una traducción incompleta pues sólo incluye el tercero de los tres ensayos que componen el volumen original, aparece firmada con las

\footnotetext{
${ }^{7}$ En otro lugar, este mismo autor (2013a: 164) apunta "Wallace even attempted to convince his scientific colleagues that spiritual forces were undetectable by scientific means because the technology had not yet been devised".
} 
iniciales J.A.M. y, según consta en la edición española, “deja bastante que desear respecto á su estilo" (Wallace, 1891: 36) ${ }^{8}$.

Sabemos también de la existencia de otra traducción que fue publicada por entregas en el periódico mexicano El Diario del Hogar. Dicha traducción está plagada de erratas de imprenta según se indica en el prólogo de la edición español ${ }^{9}$. Esta desafortunada circunstancia propició que el mismo periódico, para subsanar las deficiencias, editara de nuevo el texto mediante un sobretiro en dos tomitos titulados Los milagros y el espiritualismo moderno y Defensa del espiritismo moderno respectivamente. No tenemos conocimiento del responsable de esta traducción.

En el mismo prólogo, por otro lado, se nos informa de una versión castellana impresa en México a cargo de Alfonso Herrera, un destacado y estimado científico mexicano que abrazó el espiritismo. El texto fue remitido a España, a petición del traductor, por el presidente de la "Sociedad Espírita Central Mejicana”, el general D. Refugio I. González (Wallace, 1891: 24-25).

\section{La traducción de Defensa del espiritismo (1891) editada en España}

\subsection{Datos sobre la edición}

Defensa del espiritismo fue publicada por primera vez en España en 1891 por el Centro Barcelonés de Estudios Psicológicos y constituye el primer volumen de la colección Biblioteca Universal Espiritista. La traducción de la edición española es, según acabamos de señalar, obra de Alfonso Herrera, había sido impresa en México y se ajusta a la segunda edición inglesa, publicada en Londres el año 1881 con el título de $O n$ Miracles and Modern Spiritualism.

\footnotetext{
${ }^{8}$ Una versión del texto con la ortografía modernizada se puede encontrar en la siguiente dirección en línea: https:/espiritismo.es/Descargas/libros/defensa-del-espiritualismomoderno.pdf.

${ }^{9}$ Es posible acceder a algunas páginas de este diario a través del catálogo en línea de la hemeroteca de la Universidad Nacional Autónoma de México (UNAM).
} 


\subsection{Datos sobre el traductor}

Alfonso Herrera Fernández (1838-1901), hombre modesto, de carácter afable e ideas liberales, fue una figura clave en el desarrollo de las ciencias en México, su país natal. Tras cursar un año de medicina obtuvo el título de farmacéutico, profesión que ejerció en conjunción con otras ocupaciones entre las que destaca la de profesor, la cual, según el criterio de Justo Sierra, desempeñó de forma ejemplar: "un profesor que difícilmente puede ser reemplazado por su sabiduría y excelente método de enseñanza" (Guevara Fefer, 2002: 71). Además de por su labor docente, Herrera se distinguió por su actividad en las numerosas sociedades científicas a las que perteneció y por sus contribuciones en diversas revistas especializadas así como en otras de carácter más divulgativo. Entre sus publicaciones se encuentran algunas traducciones, un dato de interés para nuestro estudio y que no ha de resultar extraño si tenemos en cuenta que en México, en esa época, la traducción era una práctica habitual entre aquellos con acceso a la cultura (Lafarga y Pegenaute, 2013: 264). Su labor como escritor fue reconocida por la Academia Mexicana de la Lengua que lo nombró miembro honorario el 17 de septiembre de 1877. Este reconocimiento constituye un importante aval de su dominio de la lengua. Añadamos, también, que fue un hombre que realizó una gran labor filantrópica.

Un último aspecto que consideramos pertinente señalar es su vertiente espiritista. Agustin Aragón afirma, "El Sr. Herrera, sucesor del Sr. Barreda no era positivista por su principios filosóficos, era lo diametralmente opuesto: un espiritista" (Vera y Cuspinera, 2015:342). TorresSolanot (1891:25), por su parte, señala el año 1888 como fecha de la adhesión definitiva de Herrera a la doctrina y nos informa de que la misma se realizó tras un "detenido estudio y experimentación científica”. Añade que desde entonces el científico mexicano se convirtió en un entusiasta propagandista de la doctrina.

\subsection{Estructura}

Al cotejar el texto de la edición española con el texto origen (TO) se puede constatar que la macroestructura de ambos textos presenta algunas diferencias, siendo la más llamativa la incorporación en la edición española de un extenso prólogo firmado por el Vizconde de Torres- 
Solanot, uno de los más notables propagadores de la doctrina espiritista en nuestro país. Igualmente es importante reseñar la inclusión de una página con apuntes sobre la traducción del texto realizada al español así como de una fe de erratas al final de la publicación.

Ahora bien, si nos centramos en el texto de la obra de Wallace en sí, observaremos que no hay diferencias significativas en relación a las distintas partes que conforman el volumen. Ambas ediciones constan de los mismos elementos: un prefacio a cargo del autor británico, una página con citas de célebres científicos, el texto de cada uno de los tres ensayos, un apéndice y un índice. Con todo, se pueden detectar algunas diferencias que atañen a la estructura, siendo la más notoria la secuencia de los epígrafes que componen cada uno de los ensayos. En la versión original se presentan de forma consecutiva, mientras que en la versión española cada uno de los epígrafes que conforman el ensayo comienza en una nueva página. El resultado es que la versión española posee un mayor número de páginas y es ligeramente menos compacta. Esta nueva disposición de la información de la edición española se puede atribuir a un propósito de facilitar la lectura del texto (si la misma es responsabilidad del traductor o se debe a una decisión editorial queda por determinar).

La asimetría entre ambas versiones, no obstante, se hace más acusada al fijar la atención en la segmentación de los párrafos dentro de cada uno de los ensayos. El traductor, salvo en muy contadas ocasiones en que se abstiene, realiza una mayor segmentación dentro de los epígrafes sobre todo en relación a los dos últimos. Esta actuación es más patente en aquellos epígrafes cuyo propósito es ofrecer un testimonio, lo cual entendemos no es fortuito dado que la inclusión de este tipo de información -los testimonios- constituye un argumento vital en la defensa de la doctrina.

\subsection{Elementos paratextuales ${ }^{10}$}

\subsubsection{Título de la obra, título de los ensayos y título de los epígrafes}

En la edición española de la obra se opta por utilizar el título de uno de los tres ensayos "A Defence of Modern Spiritualism" para dar título al libro, con la particularidad de que se omite el artículo indefinido y el

\footnotetext{
${ }^{10}$ Entendemos el concepto paratexto según la definición de Genette (1997).
} 
adjetivo del TO. La justificación de esta elección la encontramos en el prólogo que precede al texto de Wallace, donde se apunta al respecto: "se ha preferido para la publicación de la Biblioteca, porque expresa mejor el objeto del libro y se acomoda á la nomenclatura aquí adoptada de «Espiritismo» en vez de «Espiritualismo» (Spiritualism), que usan los ingleses y los norteamericanos" (Torres-Solanot, 1891: 24). En lo relativo a los títulos de los ensayos, los títulos del primer y el tercer ensayo del volumen se han traducido de manera literal aunque, en el primer caso, omitiendo el articulo indefinido "a/an" al comienzo del mismo, una omisión que es atribuible a las normas de uso de nuestra lengua. En cuanto al segundo, se traslada alterando el orden de presentación de los elementos. En lo que se refiere a la traducción de los epígrafes, en mayúsculas tanto en TO como en el texto meta (TM), el traductor realiza una buena adaptación de los mismos.

\subsubsection{Página con citas}

Esta página, ubicada antes del prefacio en el TO y tras el prefacio en el $\mathrm{TM}$, constituye el primer contacto del autor con el lector. Contiene cinco citas, cuidadosamente seleccionadas, que pertenecen a estos cuatro ilustres científicos: Humboldt, Sir Humphry Davy, Sir John Herschell y Professor Huxley. Su contenido es una clara alusión al método científico: experimentalidad, mente abierta y libre de prejuicios, y necesidad de cuestionar teorías ya aceptadas y sirve, a nuestro parecer, para justificar su posición sobre el tema a tratar. El autor nos deja claro que conoce el procedimiento científico y nos da a entender que ha sido a través de éste como ha llegado a descubrir y abrazar la teoría de la que nos va a hablar en el libro. En otras palabras, nos dice en clave de citas cómo hay que leer la obra. Incluimos a modo de ilustración la cita de Humboldt:

A presumptuous skepticism that rejects facts without examination of their truth, is, in some respects, more injurious than unquestioning credulity. Humboldt.

Un presuntuoso escepticismo que rechaza los hechos sin examinar su realidad, es, en ciertos casos, más nocivo que la ciega credulidad. Humboldt. 


\subsubsection{Prefacio}

Este prefacio de poco más de tres páginas de extensión y firmado por el autor resulta de interés por un doble motivo: uno porque nos sirve para ilustrar algunas de las funciones de este tipo de paratextos; otro porque es el primer espacio donde de forma clara apreciamos la presencia del traductor y sirve, por tanto, para anticipar algunos de los principales rasgos de la traducción de Alfonso Herrera, a los cuales prestaremos atención en un epígrafe posterior. Por lo que respecta a la función del texto, el autor, Wallace, prepara al lector para la lectura del libro informándole de manera sucinta pero clara del tema principal del mismo: el cómo y el porqué de su adhesión y su defensa del espiritualismo. Tras informar brevemente sobre el origen y la finalidad de cada uno de los ensayos que componen el libro, el autor, en modo de narración en primera persona, nos ofrece una explicación de su adhesión al espiritismo en respuesta a las muchas críticas recibidas de parte de algunos colegas y concluye manifestando su convencimiento sobre la validez de la doctrina espiritualista ${ }^{11}$.

\subsubsection{Prólogo-Bosquejo de un prólogo}

Este texto, con el título "Importancia del Espiritismo", está etiquetado como Bosquejo de un Prólogo y aparece firmado por el vizconde de Torres-Solanot. Dado su contenido y su extensión, ocupa 27 páginas, resulta igualmente apropiado para discurrir la función de este tipo de paratexto concreto y sobre su incidencia en la lectura del texto. Con este fin vamos a proceder a apuntar unos datos sobre su autor y, seguidamente, sobre el contenido del escrito.

Antonio Torres-Solanot y Casas (1840-1902), hombre culto y descendiente de una familia noble de tradición liberal y republicana, fue un destacado defensor y propagador del espiritismo kardeciano en nuestro país. Figura destacada del espiritismo español, ocupó la vicepresidencia del Centro General del Espiritismo en España y fue presidente del primer Congreso Internacional Espiritista de Barcelona. Escribió nu-

\footnotetext{
${ }^{11}$ A nuestro entender el texto del prefacio, junto con la página de citas, ilustra lo que en opinión de Genette es la principal función de un prefacio "to get the book read and get the book read properly" (Genette, 1997: 197).
} 
merosos textos de contenido espiritista y fue editor de varias revistas afines a estas cuestiones. Su labor propagandística, afirma Mülberger (2016: 62-63), "consistió, sobre todo, en plantear y resumir de manera didáctica las bases esenciales del credo espiritista". Fue, además, según esta autora, una persona que gozó del aprecio de sus contemporáneos debido a su cultura y a sus dotes de excelente organizador. Era, por tanto, un aval para la difusión de la doctrina.

El contenido del escrito, salvando unas páginas en las que se pone en valor el Centro Barcelonés de Estudios Psicológicos y su Biblioteca, y en las que se aportan algunos datos sobre esta y otras ediciones mexicanas de la obra a las que ya nos hemos referido, se centra en describir la doctrina y atraer adeptos a la misma.

Nos presenta una doctrina de soluciones prácticas que aspira a conocer la verdad y practicar el bien. Nos explica "lo que es" y "lo que no es" y aquello que la diferencia de las religiones positivas. Nos previene de posibles ataques que podemos sufrir al adoptar la doctrina y nos anima a vencer los obstáculos que se puedan presentar. Igualmente, nos hace saber que profundizar en el espiritismo es entrar en "un mundo nuevo donde se hallan resueltas las tres eternas preguntas, aspiración del verdadero saber humano: «¿Quién soy?», «¿De dónde vengo?», «¿Adónde voy?»" (pp. 18-19). Nos facilita, además, parte del ideario de Kardec (pp. 14-18), así como un fragmento de una obra de autoría propia en defensa de la doctrina. A lo ya expuesto, añade una reseña del espiritismo moderno y ofrece los testimonios de numerosos científicos, amén de otras figuras ilustres que se han manifestado a favor de la doctrina, entre los que destaca a Alfred Russel Wallace y William Crookes ${ }^{12}$. Para finalizar, Torres-Solanot destaca las virtudes de la doctrina espiritista y manifiesta su deseo y confianza en que se imparta su enseñanza de forma oficial aduciendo la innegable importancia del espiritismo. Finaliza recomendando la lectura de los volúmenes de la Biblioteca convencido de que sus lectores agradecerán la invitación en el porvenir de los beneficios que ésta les reportará.

Hay que hacer notar que muchas de estas consideraciones aparecerán también en la obra de Wallace (las posibles críticas, la aportación de

\footnotetext{
${ }^{12}$ Roberto Hare, doctor en Medicina y distinguido profesor de Química de la Universidad de Pensilvania y los astrónomos Liais y Flammarion son otros de los nombres que se mencionan.
} 
testimonios de personas ilustres, la postura del autor), por lo que pensamos que el prólogo de Torres-Solanot, al dar cabida a estas informaciones, prepara al lector para la lectura de la obra creando un marco idóneo para la misma y sirve de ilustración de un prólogo mediador entre el texto y el lector. Por su contenido y su forma de expresión, el prólogo del Vizconde constituye todo un reclamo para leer el libro y adherirse a la doctrina espiritista. En suma, crea un marco idóneo para la lectura de la obra de Wallace, ayudando a que el texto halle su lugar en un nuevo espacio cultural, aquel de la España de finales del siglo XIX.

\subsubsection{Página informativa sobre la traducción}

Se trata de un paratexto sin título en el que se informa sobre una traducción de la obra de Wallace publicada en Santiago de Cuba ${ }^{13}$ así como sobre los textos de referencia que el traductor de la obra que se presenta ha empleado en el desempeño de su labor. Por una parte, el original en inglés de la segunda edición y, por otra, y de manera complementaria, se ha tenido a la vista la edición alemana traducida por Gregor Constantin Wittig, y adicionada con algunos comentarios (Leipzig, 1875) ${ }^{14}$. No se proporciona información sobre el método de traducción empleado en relación al proceder del traductor. Se indica tan sólo que es obra del Sr. Herrera y se valora el producto -es correcta y completa-, justificando así su ser y estableciendo de este modo una distancia con la citada traducción a cargo de J.A.M que se juzga estilísticamente inadecuada (Wallace, 1891: 36).

\subsubsection{Notas a pie de página}

En esta edición las notas a pie de página tienen un triple origen. Unas proceden del autor, otras del traductor y unas terceras de la Biblioteca. Las primeras, las del autor, marcadas con un asterisco en el TO suman diecinueve en total. Todas, independientemente de su extensión (algunas son realmente extensas, ver notas de las páginas 181 y 190), son

\footnotetext{
${ }^{13}$ Hemos hecho referencia a esta obra en el epígrafe 3 de este estudio.

${ }^{14}$ Llama la atención que se hiciera uso de una versión en alemán cuando es conocido que la lengua que gozaba de gran prestigio en México en el siglo XIX era el francés. Conviene saber que el año de publicación de la primera traducción gala de la obra es 1891.
} 
respetadas por el traductor a excepción de una que, a nuestro entender, es prescindible para el lector de la obra en nuestro idioma. Se trata de la nota de la página 212 donde el autor informa de que el párrafo donde se hace la llamada de cita se había omitido en una publicación anterior del ensayo. El resto de las notas, por el contrario, sí pueden considerarse relevantes ya que complementan la información del cuerpo del texto a la vez que pueden ayudar al lector a una mejor comprensión del mismo. La mayoría de las veces el autor, haciendo uso de distintas fuentes, las emplea para aportar argumentos o testimonios a favor de su posición y para rebatir y criticar aquellos que son contrarios a su postura. Otras, las menos, las emplea para añadir una precisión a un dato concreto.

Las segundas, las del traductor, son diez y están claramente diferenciadas de las anteriores. Todas, a excepción de un par que aparecen señalizadas con todas las letras, están indicadas mediante la abreviatura N. de T., la cual suele aparecer entre paréntesis. En la mayor parte de ellas el traductor interviene, dándonos cuenta de su formación y conocimiento sobre el tema, para ofrecer una puesta al día de la información sobre el espiritismo. En otras participa para definir o explicarnos una palabra (notas de las páginas 158 y 258). Encontramos también unas terceras notas en las que decide participar para, de forma muy explícita, establecer nexos entre la cultura origen y la cultura meta (entendiendo México e hispanohablantes). Aludiendo a un ejemplo de esta última, el traductor acerca lo expuesto en el TO al lector y facilita así la comprensión del mismo. Incluimos a continuación tres ejemplos a modo de ilustración:

En la actualidad estas cifras han aumentado extraordinariamente: poblaciones enteras son espíritas, como sucede con Onced, cerca de Boston. (N. del T.), p. 250.

Se designa con este nombre (se refiere a "levitación") el hecho de que se eleve en el aire cualquier cuerpo sin la intervención de ninguna de las fuerzas conocidas. (N. del T.), p. 258.

En el año de 1883 estuvo en la capital de Méjico la Sra. Altagracia Ojeda que posee la facultad de la doble vista bastante desarrollada. Leia con facilidad cualquier escrito que se le presentaba, teniendo los ojos perfectamente vendados con un lienzo ó cerrados y comprimidos los párpados por los dedos del observador, ó envuelta completamente con una tela negra de tejido apretado. Se hicieron varios experimentos en presencia de hombres científicos, periodistas y multitud de personas; la 
prensa diaria de la capital refirió con exactitud los fenómenos observados, á los que por desgracia no se les dió la importancia, que se merecen esta clase de hechos. (N. del T.), pp.135-136.

Un comentario aparte, dada su extensión, merece la nota que ocupa la página 165, donde el traductor ofrece una lista inconclusa de personas distinguidas que son "espíritas".

El tercer tipo de notas, las Notas de la Biblioteca, también se encuentran debidamente indicadas. Suman cinco, están señaladas con la abreviatura N. de la B. y tienen, en nuestra opinión especial relevancia porque son las únicas dirigidas al lector español. Se crean para explicar o precisar el uso de términos y para incidir en el carácter universal de la doctrina a la vez que manifestar una puesta al día en la información. En este sentido destaca la incluida en las páginas 108-110, donde se aporta una lista de publicaciones periódicas espiritistas elaborada a fecha de abril de 1891. Estas notas complementan, de alguna manera, aquellas del traductor. He aquí dos ejemplos:

El autor se fija en la fecha señalada para indicar la divulgación del Espiritismo en América, pero ya antes habían ocurrido los primeros hechos que llamaron la atención. Véase lo dicho en el Prólogo. (N. de la B.), p. 242.

Extasis sonambúlico en los médiums. (N. de la B.), p. 360, (la llamada de cita corresponde al vocablo "trance")

\subsubsection{Apéndice}

Esta sección aparece dividida en cuatro apartados bien diferenciados mediante números romanos (I-IV), los cuales, en nuestra opinión, cumplen la misma función que las notas del autor: complementar la información del texto de la obra. Constituye, junto con el espacio de algunas de las notas del autor, el lugar donde Wallace incorpora esa información complementaria de la que habla al comienzo del prefacio y que considera precisa tras haber revisado el texto de los artículos que componen la obra: "Considerable additions have been made of illustrative fact, argument, and personal experience, together with a few critical remarks on Dr. Carpenter's latest work" (Wallace, 1875: v). 


\subsubsection{Erratas}

En este espacio se apuntan cinco erratas con indicaciones sobre la página y la línea donde se pueden localizar. La inclusión de una fe de erratas al final del libro es una muestra de que el texto ha sido revisado ${ }^{15}$.

\subsection{La traducción y los procedimientos de traducción ${ }^{16}$}

En una primera valoración apuntaremos que la traducción del texto a cargo de Alfonso Herrera constituye un ejemplo de traducción de forma directa del inglés. Pese a que se ha tenido a la vista la edición alemana traducida por Gregor Constantin Wittig, no hemos detectado la mediación de esta versión, la cual es bastante literal respecto a la obra original, en la traslación del texto. En términos generales la traducción de Herrera puede describirse como una versión íntegra y respetuosa con el original además de estilísticamente acertada. El traductor, a nuestro juicio, trata de reproducir con fidelidad el mensaje del texto origen en la lengua receptora de la forma más natural posible en aras a acercar el mismo al lector. Una finalidad que, en última instancia y dado su perfil espiritista, suponemos motivó que asumiera la responsabilidad de verter el texto al castellano. Esta voluntad difusora se deja notar en diversos procedimientos a los que recurrió al verter la obra a nuestro idioma. La actitud de respeto al texto no ha evitado, sin embargo, que sintamos su presencia cuando lo leemos. Describimos a continuación algunos de los procedimientos de su actuación.

\subsubsection{Elementos ortotipográficos}

Cuando se cotejan el TO y el TM se pueden apreciar algunas diferencias en el uso de estos elementos y ello a pesar de que el traductor actúa en términos generales de forma respetuosa. Estas discordancias en muchos casos obedecen a las exigencias normativas de uso de la lengua meta así como, pensamos, a un deseo de facilitar la lectura del texto. Pero en

\footnotetext{
${ }^{15}$ A pesar de ser una traducción revisada, se pueden encontrar algunos errores (descuidos), algunas equivalencias léxicas inexactas o dudosas y, ocasionalmente, alguna oración difícil de comprender.

${ }^{16}$ Hacemos uso de la terminología empleada por Hurtado Albir (2001).
} 
otros carecen de justificación normativa y solo se pueden explicar como resultado de una intervención (más o menos) deliberada del traductor y como tal no está exenta de significado.

Así en el TM encontramos en cursiva palabras y fragmentos que no están de esta manera señaladas en el TO y viceversa, palabras y fragmentos que se encuentran indicadas de ese modo en el TO y el traductor las deja sin marcar. Una situación muy similar acontece en relación al uso de las comillas. Ejemplos:

very word "supernatural," as applied to a fact, is an absurdity p.37 \# La palabra sobrenatural aplicada á un hecho es absurda, p.93.

the logical conclusion from the facts was, that it was the spirit* of the murdered man;p.147 \# se dedujo lógicamente que el espíritu del hombre asesinado era en efecto el que los causaban, p.244.

"modern spiritual manifestation" p.147 \# manifestación moderna de los espíritus, p. 242.

the partial solution of the most difficult of all problems -the origin of consciousness, and the nature of mind. P107 \# la resolución del más difícil de los problemas, el origen de la conciencia y la naturaleza del espíritu, p.188.

Con respecto a los nombres propios que se mencionan, especialmente los antropónimos, el traductor, en general, actúa manteniendo la forma original salvo en aquellos casos en los que existe una adaptación de los mismos ya arraigada en nuestra lengua. En el resto de los casos, los deja inalterados aunque pueden observarse, no obstante, algunos descuidos ${ }^{17}$. El mismo proceder manifiesta cuando se trata de los nombres de revistas o títulos de libros, que pasan al texto terminal igual que se encuentran en el original. En estos últimos casos, en ocasiones, el traductor también incluye la traducción del libro o la revista entre paréntesis.

\subsubsection{Elisión y compresión, ampliación y amplificación}

No son procedimientos de traducción que distingan la labor traslativa del traductor ni tampoco que se puedan detectar con facilidad. Aun

\footnotetext{
${ }^{17}$ Así encontramos Miss Catalina Fox en la p. 243 y Miss Kate Fox en la p. 257.
} 
así se pueden encontrar algunos ejemplos, especialmente de elisiones, salpicadas a lo largo de la obra. La mayor parte de las mismas apenas inciden en la lectura del texto salvo para facilitar su lectura y no afectan de manera decisiva al significado pero otras, las menos e independientemente de su extensión, sí pueden tener un efecto sobre el mensaje adulterándolo ligeramente y/o confiriendo al texto un carácter más taxativo: La elisión de "partial" en el último ejemplo del epígrafe anterior sirve de ilustración al igual que la omisión de la pregunta: "And what have we per contra?" al final de un párrafo en la página 238 del texto.

Por otra parte, en lo que respecta a las ampliaciones y amplificaciones, el número de ejemplos encontrados se restringe a casos de amplificaciones y es poco significativo. Entendemos que son una variante estilística para aportar mayor claridad a una determinada unidad informativa (The Vedas, p.177 / los libros de los Vedas, p.287).

\subsubsection{Variaciones estilísticas}

$\mathrm{Al}$ aproximarnos al texto hemos hallado algunas diferencias estilísticas que pensamos que derivan del deseo de adaptar el texto a nuestra lengua y/o hacer la lectura más fácil. Así se pueden encontrar fragmentos donde se aprecian modificaciones en el orden de presentación de los componentes de una oración o variaciones en el uso de los tiempos verbales.

He also supposes that in accepting the spiritual doctrines I have been to some extent influenced by clerical and religious prejudice, p.vi.

Supone tambien que por las preocupaciones ${ }^{18}$ religiosas acepto la doctrina espiritualista, p.38.

I am surely following a strictly logical and scientific course p.vii.

Estoy seguro de que he seguido un método extrictamente científico, p.39.

\footnotetext{
${ }^{18}$ Consideramos pertinente señalar, tras consultar el Nuevo Tesoro Lexicográfico de la Lengua Española (NTLLE), una de las acepciones del vocablo preocupación recogida en el Diccionario de la Real Academia Española, en su edición de 1884: "Juicio ó primera impresión que hace una cosa en el ánimo de uno, de modo que no le permite admitir otras especies ó asentir á ellas”, p. 860,2.
} 
En ocasiones el traductor se distancia del TO asumiendo en primera persona lo que el autor expresa con fórmulas más impersonales estableciendo así una menor distancia con el lector p.ej. It is worthy of remark p.147/ Debo notar p.243; siendo más asertivo: We may therefore conclude, p.144/ podemos asegurar p.239; o realizando una afirmación donde en el texto hay formulada una pregunta:

but is it so illogical when these noises turn out to be signals, and signals which spell out a fact, which fact, though wholly unknown to all present, turns out to be true?, p.147.

pero es ilógico cuando se convierten en señales que dan á conocer hechos ignorados por todos los presentes y que después se comprueba que son ciertos, p.243.

En suma, y por lo que concierne a la intervención del traductor, nos encontramos a un traductor que omite palabra(s), a un traductor que interviene para alterar el orden de los elementos de una frase o realizar un cambio en el uso del tiempo del verbo, y a un traductor que a veces se permite modificar el tono y el modo de expresión empleado por el autor. También a un traductor culto (uso del vocablo "anfibológica") y a un traductor con recursos a la hora de traducir (Facts, however, are stubborn things p.7 / Pero los hechos son muy elocuentes p.39). Pero, por encima de todo, un traductor comprometido ideológicamente con la obra. Sirva de ilustración de su actuación el fragmento con el que el autor concluye el prefacio y que reproducimos a continuación (el subrayado y el uso de mayúsculas es nuestro).

-I am surely following a strictly logical and scientific course, in seeing how far this doctrine will enable us to account for some of those residual phenomena which Natural Selection alone will not explain. In the 10th chapter of my Contributions to the Theory of Natural Selection I have pointed out what I consider to be some of these residual phenomena; and I HAVE SUGGESTED that they may be due to the action of some of the various intelligences above referred to. This view was, however, put forward with hesitation, and I myself suggested difficulties in the way of its acceptance; but I maintained, and still maintain, that it is one which is logically tenable, and is in no way inconsistent with a thorough acceptance of the grand doctrine of Evolution, through Natural Selection, although implying (as indeed many of the chief supporters of that 
doctrine admit) that it is not the all-powerful, all-sufficient, and only cause of the development of organic forms, pp.vii-viii

Estoy seguro de que he seguido un método extrictamente científico para llegar al establecimiento de esta proposición: por la doctrina espiritualista se explican ciertos hechos, cuya causa no es posible elucidar por medio de la Selección natural. En el Capítulo X de mi obra intitulada "Contributions of Natural Selection" he indicado algunos de esos fenómenos, Y HE MANIFESTADO como se pueden explicar por la acción de los séres inteligentes ya mencionados. Sin embargo, emití esta opinión de una manera anfibológica, y expuse yo mismo las objeciones á que estaba sujeta. Pero desde que me convencí de la verdad del espiritualismo he sostenido que esta doctrina es la única que puede dar la explicación de ciertos fenómenos, sin ser por esto contraria á la gran teoría de la Evolución por medio de la Selección Natural, pp.39-40.

\section{Comentario final}

El texto de la edición española de la obra, Defensa del espiritismo (1891), de A. R. Wallace es una traducción importada desde México, país donde la obra se había publicado con anterioridad. Constituye uno de los testimonios del interés que se despertó en nuestro país por creencias alternativas a aquella de la religión católica, entre ellas el espiritismo. Este fenómeno, aunque no al unísono, se produjo en otros muchos países geográfica y no geográficamente cercanos, durante el último tercio del siglo XIX. La edición española de la obra, a diferencia de la editada con anterioridad en México, resulta particularmente interesante por la inclusión de un prólogo, por encargo de la editorial, de D. Antonio Torres-Solanot, uno de los más notables propagadores de la doctrina espiritista en España. La redacción de este prólogo seguramente intervino en la recepción que la obra tuvo en nuestro país e ilustra el, a veces subestimado, papel mediador de la editorial. En el referido texto, el autor nos ofrece, como no puede ser de otra manera, dada su trayectoria vital y su ideario, una visión tendenciosa, pero, a su vez, argumentada, a favor de la plena aceptación de la doctrina.

El traductor, Alfonso Herrera, al igual que el autor del libro, fue un célebre naturalista en su país así como un seguidor y difusor de la creencia espiritista, doctrina que, al igual que el autor, abrazó tras meticuloso estudio y experimentación científica sobre la misma. Creemos poder afirmar, por tanto, que conocía al autor y conocía, y además en 
profundidad, el tema. Tenía, además, experiencia como autor de artículos y también, aunque presumimos que en menor medida, como traductor. Su labor como escritor es difícilmente cuestionable ya que ostenta la distinción de ser miembro honorario de la Academia Mexicana de la Lengua desde 1877.

El texto se vierte directamente del inglés y se ajusta, tal y como se apunta en el prólogo, a la segunda edición inglesa de la obra de Wallace. Es una traducción completa pero que se aleja de la literalidad y, en consecuencia, presenta algunas diferencias que, en términos generales, no alteran el significado del texto original. Entendemos que en las decisiones del traductor prima un deseo de adaptar el texto para lograr una mejor y mayor recepción del mismo por parte del lector (de origen mexicano primordialmente), una aspiración que difícilmente se puede cuestionar si se tiene en cuenta el tipo de texto a traducir, un ensayo filosófico-religioso sobre un tema por el que él mismo siente una fuerte inclinación y, suponemos, desea difundir.

\section{Bibliografía}

Bastin, Georges L. 2011. Traductores comprometidos con la independencia: el caso venezolano. Revista Historia y Sociedad 20: 33-55.

Berry, Andrew. 2013a. Alfred Russel Wallace: Evolution's red-hot radical. Nature 496: 162-164.

Berry, Andrew. 2013b. Alfred Russel Wallace - natural selection, socialism, and spiritualism. Current Biology 23:24 pR1066-R1069.

De Mateo Avilés, Elías. 2011. Espiritistas y teósofos en Andalucía (18531935). Málaga: Editorial Sarriá.

Costa, J. T. 2014. Wallace, Darwin, and the Origin of Species. Harvard: Harvard University Press.

Genette, Gerard. 1997. Paratexts. Cambridge University Press.

Ginnobili, S. \& Blanco, D. 2017. Wallace's and Darwin's natural selection theories. Synthese. doi:10.1007/s11229-017-1491-z.

Guevara Fefer, Rafael. 2002. Los últimos años de la historia natural y los primeros días de la biología en México. Universidad Nacional Autónoma de México.

Hurtado Albir, Amparo. 2001. Traducción y traductología: introducción a la traductología. Madrid: Cátedra.

Lafarga, Francisco \& Pegenaute, Luis (eds.). 2013. Diccionario histórico de la traducción en Hispanoamérica. Madrid: Iberoamericana/Vervuert 
Mülberger, Annette (ed.). 2016. Los límites de la ciencia: espiritismo, hipnotismo y el estudio de los fenómenos paranormales (1850-1930). Madrid: Editorial CSIC Consejo Superior de Investigaciones Científicas.

Penalva Mora, Vicente. 2013. El orientalismo en la cultura española en el primer tercio del s. XX. La sociedad teosófica española (1888-1940). (Tesis doctoral). Universidad Autónoma de Barcelona. Departamento de Historia Moderna y Contemporánea.

Revets, S. A. 2009. Alfred Russel Wallace, co-author of the Darwin-Wallace Theory of Evolution. Journal of the Royal Society of Western Australia, 92: 369-372.

Smith, Charles H. 2009. What's in a Word? On Reading-and Misreading-Alfred Russel Wallace. The Linnean, special issue 9: 35-44.

Vera y Cuspinera, M. ${ }^{a}$ Eugenia. 2015. La ciencia cuestionada. Espiritismo versus Positivismo en el siglo XIX mexicano (Tesis para optar al grado de Doctora en Filosofía). Universidad Nacional Autónoma de México.

Verdejo Segura, M. ${ }^{a}$ del Mar. 2011. La traducción como instrumento de difusión de creencias protestantes: el caso de Fundamentos de la historia de Manrique Alonso Lallave. En Juan Jesús Zaro Vera (ed.). La traducción como actividad editorial en el siglo XIX. Sevilla: Ediciones Alfar, 185232.

Wallace, Alfred Russel. 1875. On Miracles and Modern Spiritualism. Londres: James Burns.

Wallace, Alfred Russel. 1891. Defensa del espiritismo. Barcelona: Imp. de Redondo y Xumetra.

Zaro Vera, Juan Jésus. 2015. Traducción y propaganda religiosa: Gibraltar y la labor traductora de William Harris Rule. Quaderns. Revista de Traducció 22: 135-148. 
